\title{
Second États Des Lieux Des Facteurs De Décompensation Cétosique Du Diabète Au Centre National Hospitalier Universitaire De Cotonou
}

\author{
Comlan Jules Gninkoun (MD.) \\ Ogountchoro Edwige
}

Département de Médecine et Spécialités Médicales, Faculté des Sciences de la Santé, Université d’Abomey Calavi

\section{Diallo Mamadou Mansour}

Service d'Endocrinologie Diabétologie Hôpital Donka CHU de Conakry, Guinée

\section{Alassani Adébayo}

Département de Médecine et Spécialités Médicales,

Faculté de Médecine, Université de Parakou, Bénin

\section{Diallo Alpha Mamadou}

Service d'Endocrinologie Diabétologie Hôpital Donka CHU de Conakry, Guinée

\section{Doi:10.19044/esj.2021.v17n29p173}

Submitted: 31 March 2021

Accepted: 24 June 2021

Published: 31 August 2021
Copyright 2021 Author(s)

Under Creative Commons BY-NC-ND 4.0 OPEN ACCESS

Cite As:

Gninkoun C.J., Edwige O., Mansour D.M., Adébayo A. \& Mamadou D.A. (2021). Second États Des Lieux Des Facteurs De Décompensation Cétosique Du Diabète Au Centre National Hospitalier Universitaire De Cotonou. European Scientific Journal, ESJ, 17(29), 173.

https://doi.org/10.19044/esj.2021.v17n29p173

\section{Résumé}

Introduction: Le diabète est une pathologie métabolique chronique en pleine expansion dans le monde. La décompensation cétosique constitue l'une de ses complications métaboliques qui dépend de plusieurs variables. L’objectif de ce travail était de décrire les facteurs de décompensations cétosiques du diabète chez des patients hospitalisés au CNHU de Cotonou.

Méthode: Il s’agit d'une étude rétrospective, descriptive et analytique qui s’est déroulée du 1er Juin 2016 au 31 Mai 2019. Elle a porté sur les patients diabétiques hospitalisés dans le service d'endocrinologie au cours de la 
période d'étude et ayant une hyperglycémie supérieure ou égale à 2,5 g/l, une cétonurie au moins égale à deux croix associée à une glycosurie positive.

Résultats: Au total, 196 patients ont été inclus. L’âge moyen était de 43,73 ans $\pm 16,2$ ans. On notait une légère prédominance féminine avec un sex ratio de 0,960,96. La prévalence des décompensations cétosiques était de 28,99\%. Les principaux facteurs de survenue des décompensations cétosiques étaient les infections dans 67,86 \% des cas et l'inobservance dans $29,59 \%$ des cas. Les foyers infectieux les plus fréquents étaient génito-urinaires dans 23,30 $\%$, respiratoires dans 18,04 \% et le paludisme dans 32,33 \% des cas. Le sexe féminin était statistiquement plus souvent associé aux infections urogénitales.

Conclusion: Les principaux facteurs de décompensation n'ont pas évolué et demeurrent de façon prépondérante les infections. L'étude des determinants de ces infections pourrait servir à une prévention plus ciblée.

Mots-clés: Diabète, Cétoacidose, Infections, Complications Aiguës

\title{
Second Status Report Of Diabetes Ketoacidosis Precipitating Factors At The Centre National Hospitalier Universitaire De Cotonou
}

\section{Comlan Jules Gninkoun (MD.) \\ Ogountchoro Edwige}

Département de Médecine et Spécialités Médicales, Faculté des Sciences de la Santé, Université d’Abomey Calavi

Diallo Mamadou Mansour

Service d'Endocrinologie Diabétologie Hôpital Donka CHU de Conakry, Guinée

\section{Alassani Adébayo}

Département de Médecine et Spécialités Médicales,

Faculté de Médecine, Université de Parakou, Bénin

\section{Diallo Alpha Mamadou}

Service d'Endocrinologie Diabétologie Hôpital Donka CHU de Conakry, Guinée

\begin{abstract}
Introduction: Diabetes is a chronic metabolic disease with a rapidly increasing incidence worldwide. Diabetic ketoacidosis (DKA) is one of its metabolic complications, depending on several factors. Our objective was to describe the factors that trigger DKA in patients hospitalized at the CNHU of Cotonou.
\end{abstract}


Methods: This was a retrospective, descriptive and analytical study that took place from June 1, 2016 to May 31, 2019. It focused on diabetic patients hospitalized in the endocrinology department during the study period and presenting with hyperglycemia greater than or equal to $250 \mathrm{mg} / \mathrm{dL}$, ketonuria at least equal to two crosses associated with positive glycosuria.

Results: A total of 196 patients were included. The mean age was $43.73 \pm 16.2$ years. There was a slight female predominance with a sex ratio $(\mathrm{M} / \mathrm{F})$ of 0.96 . The prevalence of DKA was $28.99 \%$. The main factors associated with DKA were infections in $67.86 \%$ of cases and non-compliance with treatment in $29.59 \%$ of cases. The most frequent infections were genitourinary in $23.30 \%$ of cases, respiratory in $18.04 \%$ of cases and malaria in $32.33 \%$ of cases. Female gender was statistically more often associated with urogenital infections.

Conclusion: The main factors associated with DKA did not change over time and remained mainly infections. The study of the determinants of these infections could be used for more targeted prevention.

Keywords: Diabetes, Ketoacidosis, Infections, Acute Complications

\section{Introduction}

Le diabète sucré constitue un véritable problème de santé notamment dans les pays en voie de développement du fait des difficultés diagnostiques et thérapeutiques [Bonkoungou $P$ et al. 2017]. Au Bénin entre 2001 et 2015, la prévalence du diabète a connu une évolution exponentielle passant de 1,1 $\%$ [Wild S et al. 2004] à 2,6 \% en 2008 [Gning SB et al. 2007] et à $12.4 \%$ en 2015 [Djrolo et al. 2015],. L’évolution du diabète sucré peut être émaillée de complications aiguës ou chroniques [Tchaou BA et al. 2014]. L'acidocétose diabétique (ACD) et le syndrome hyperglycémique hyperosmolaire (SHH) sont les complications aiguës hyperglycémiques les plus fréquentes [ Bonkoungou $P$ et al. 2017, Tchaou BA et al. 2014]. La prévalence hospitalière de ces complications aiguës est élevée dans les pays en voie de développement. En effet, en Côte d'ivoire et au Burkina-Faso la prévalence de l'ACD était respectivement de 30,7 \% [Lokrou A et al. 2014] et 59,1\% [Bonkoungou $\mathrm{P}$ et al. 2017]. C'est une complication potentiellement grave, et la mortalité par ACD varie de $14 \%$ à $50 \%$ selon les études [Bonkoungou P et al. 2017, Tchaou BA et al. 2014]. Les principaux facteurs de décompensation décrits sont dominés par les infections, la mauvaise observance thérapeutique, et la méconnaissance de la maladie [ Bonkoungou P et al. 2017, Tchaou BA et al. 2014, Kakoma PK et al. 2014]. Au Bénin, une étude réalisée dans le service d'endocrinologie du CNHU sur les décompensations cétosiques en 2009 avait permis de retrouver une prévalence des décompensations cétosiques de 21,40\%. Les principaux facteurs de décompensations étaient 
les infections dans 51,49 \% des cas, l'inobservance thérapeutique dans 25,74 $\%$ des cas et dans $24,8 \%$ des cas, aucun facteur n'a été retrouvé [Gninkoun et al. 2016]. Les foyers infectieux étaient prédominants dans cette étude.

L'objectif de ce travail était de faire une mise à jour des connaissances sur les facteurs favorisant les décompensations cétosiques chez les patients hospitalisés.

\section{Patients et méthode}

Il s’agit d'une étude rétrospective, descriptive et analytique ayant porté sur des patients diabétiques hospitalisés dans le service d'endocrinologie du Centre National Hospitalier Universitaire Hubert Koutoukou Maga (CNHUHKM) du $1^{\text {er }}$ Juin 2016 au 31 Mai 2019. Les malades diabétiques hospitalisés ayant une cétonurie, au moins égale à deux croix, associée à une glycosurie positive et une hyperglycémie supérieure ou égale à 2,5 g/L [Gninkoun et al. 2016] ont été inclus dans notre étude.

Les variables suivantes ont été collectées : l'âge , le sexe, la profession, l'ancienneté du diabète, le type de diabète, les facteurs de décompensation, l'indice de masse corporelle (IMC), la glycémie, la glycosurie, la cétonurie, la kaliémie, la natrémie, la goutte épaisse, la densité parasitaire, l'examen cytobactériologique des urines (ECBU), la radiographie pulmonaire, l'hémoculture.

En ce qui concerne les facteurs de décompensation, il a été défini deux catégories:

- les causes infectieuses qui regroupaient les foyers pulmonaires, génitourinaires, digestifs, cutanés, le pied diabétique et le paludisme

- les causes non infectieuses regroupant l'inobservance thérapeutique, la méconnaissance du diabète et les affections intercurrentes en particulier l'infarctus du myocarde et l'accident vasculaire cérébral.

Les foyers infectieux ont été définis comme suit :

La pneumonie a été retenue devant des symptômes cliniques évocateurs dont la fièvre, une douleur à type de point de côté, une toux avec expectoration, une diminution de l'ampliation thoracique, une sub-matité thoracique, l'augmentation des vibrations vocales à la palpation combinée à la percussion, diminution ou abolition du murmure vésiculaire avec râles crépitants à l'auscultation. Sur le plan paraclinique des opacités systématisées, à contours bien délimités, non rétractiles avec ou sans bronchogramme aérien à la radiographie pulmonaire.

Le foyer urinaire a été retenu devant des brûlures mictionnelles, une pollakiurie, une dysurie, des douleurs hypogastriques, un ECBU mettant en évidence une leucocyturie $\geq 10^{4}$ et bactériurie $\geq 10^{5}$. 
Le foyer génital a été retenu chez une femme devant un prurit vulvaire, des leucorrhées et l'identification du germe à la culture après prélèvement vaginal et/ou identification d'un germe à l'hémoculture.

Le paludisme a été retenu devant une fièvre, des frissons, des courbatures, des céphalées intenses, des nausées et vomissements. Sur le plan paraclinique une goutte épaisse / frottis sanguin positif.

Les données ont été collectées à l'aide d'un questionnaire électronique conçu à partir de l'application Kobocollect basée sur le système CAPI (Computer Assister Personal Interview). La source de données était les dossiers des patients diabétiques hospitalisés et le registre d’hospitalisation. La cohérence des données a été vérifiée et les données aberrantes ainsi que les doublons ont été supprimés. L'analyse a été faite à l'aide des logiciels SPSS version 21 et $\mathrm{R}$ version 3.6.1. Les mesures de fréquences ont été utilisées pour décrire et analyser les données collectées. Ainsi, la variable qualitative avait été décrite sous forme de proportions et les variables quantitatives sous forme de paramètres de position. Les comparaisons des moyennes ont été faites à l'aide du t test et celles des proportions ont été faites à l'aide du test de Khi 2 ou du test de Fisher selon le cas. Le seuil de significativité a été fixé à 0,05.

\section{Résultats}

\section{Caractéristiques de la population d'étude}

Au total 676 dossiers de patients diabétiques hospitalisés ont été dépouillés au nombre desquels ont été identifiés 196 dossiers de patients diabétiques hospitalisés pour une décompensation cétosique. Notre population était composée de 37 diabétiques de type 1 (18,87\%) ; 157 diabétiques de type 2 (80,10\%) et de 2 cas de diabète gestationnel (1,02\%). L'âge moyen était de 43,73 ans $\pm 16,2$ ans avec des extrêmes de 13 et de 100 ans. Il y avait une légère prédominance féminine avec un sex-ratio de 0,96 . La durée moyenne d'évolution du diabète était de 6,1 années.

\section{Caractéristiques des décompensations cétosiques Prévalence des décompensations cétosiques}

La prévalence des décompensations cétosiques était de 28,99 \% [25,5732,41] IC95\% .

Tableau I. Principaux facteurs déclenchant des décompensations cétosiques Cotonou 2019, $\mathrm{N}=196$

\begin{tabular}{|c|c|c|}
\hline & Effectifs (N=196) & Pourcentage (\%) \\
\hline Infection & $\mathbf{1 3 3}$ & $\mathbf{6 7 , 9}$ \\
\hline Inobservance thérapeutique & $\mathbf{5 8}$ & $\mathbf{2 9 , 6}$ \\
\hline Affection intercurrente & 06 & 3,1 \\
\hline Méconnaissance diabète & 14 & 7,1 \\
\hline Facteur non identifié & 18 & 9,2 \\
\hline Autres & 01 & 0,5 \\
\hline
\end{tabular}




\section{Facteurs déclenchant des décompensations cétosiques}

Les infections (67,9\%) et l'inobservance du traitement (29,6\%) étaient les principaux facteurs retrouvés. (Tableau I)

\section{Foyers infectieux}

Les foyers infectieux étaient principalement ceux génito-urinaires (23,3 \%), respiratoires (18\%) et le paludisme (32,3\%) (Tableau II) Tableau II: Répartition des foyers infectieux chez les patients hospitalisés pour décompensation cétosique, Cotonou 2019, $N=196$.

\begin{tabular}{|c|c|c|}
\hline Foyers & Effectifs (N=133) & Pourcentage \% \\
\hline Pulmonaire & 24 & 18,0 \\
\hline Urogénital & 31 & 23,3 \\
\hline Digestif & 05 & 3,8 \\
\hline Cutané & 20 & 15,0 \\
\hline Pied diabétique & 10 & 7,5 \\
\hline Paludisme & 43 & 32,3 \\
\hline ORL & 03 & 2,2 \\
\hline Non retrouvé & 07 & 5,3 \\
\hline
\end{tabular}

\section{Evolution temporelle des foyers infectieux}

La fréquence du paludisme était croissante pendant la période d'étude et représentait respectivement $19 \%, 21 \%$ et $37 \%$. La figure 1 nous présente l'évolution annuelle des foyers infectieux.

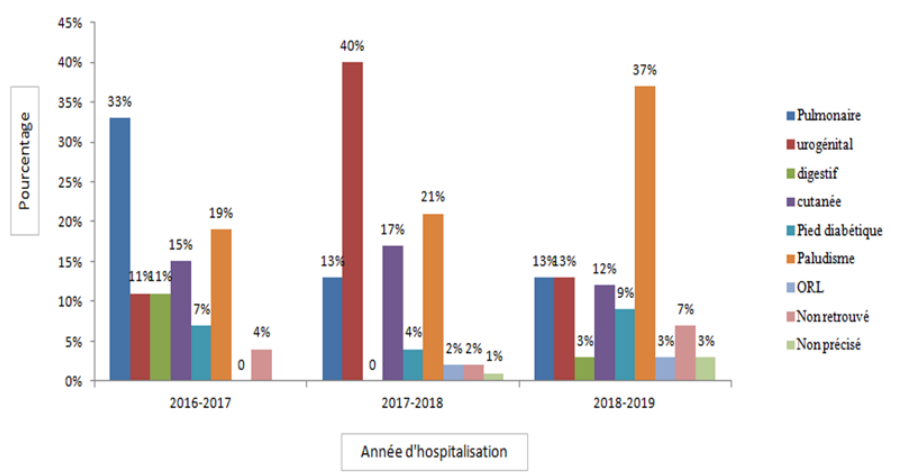

Figure 1. Evolution temporelle des foyers infectieux chez les patients admis pour décompensation cétosique Cotonou 2019, N=196

\section{Influence du sexe sur les foyers infectieux}

Il y a une association statistiquement significative entre le sexe féminin et le foyer urogénital. En effet, 23 femmes avaient une infection urogénitale soit $34,9 \%$ des cas versus 6 hommes soit 9,0 \% des cas (Tableau III) 
Tableau III. Répartition des facteurs de décompensation selon le sexe Cotonou 2019, $\mathrm{N}=196$

\begin{tabular}{|c|c|c|c|}
\hline & \multicolumn{2}{|c|}{ Sexe } & \multirow[b]{2}{*}{ p-value } \\
\hline & $\begin{array}{c}\text { Masculin } \\
\text { N (\%) }\end{array}$ & $\begin{array}{c}\text { Féminin } \\
\text { N (\%) }\end{array}$ & \\
\hline \multicolumn{4}{|l|}{ Facteurs de décompensations } \\
\hline Autres facteurs & $29(30,2)$ & $34(34)$ & \multirow[t]{2}{*}{0,678} \\
\hline Infectieux & $67(69,8)$ & $66(66)$ & \\
\hline \multicolumn{4}{|l|}{ Pied diabétique } \\
\hline Oui & $6(8,9)$ & $4(6,0)$ & \multirow[t]{2}{*}{0,744} \\
\hline Non & $61(91,0)$ & $62(94,0)$ & \\
\hline \multicolumn{4}{|l|}{ Urogénital } \\
\hline Oui & $6(9,0)$ & $23(34,8)$ & \multirow[t]{2}{*}{0,001} \\
\hline Non & $61(91,0)$ & $43(65,1)$ & \\
\hline \multicolumn{4}{|l|}{ Pulmonaire } \\
\hline Oui & $13(19,4)$ & $11(16,7)$ & \multirow[t]{2}{*}{0,853} \\
\hline Non & $54(80,6)$ & $55(83,3)$ & \\
\hline \multicolumn{4}{|l|}{ Cutané } \\
\hline Oui & $09(13,4)$ & $11(16,7)$ & \multirow[t]{2}{*}{0,780} \\
\hline Non & $58(86,6)$ & $55(83,3)$ & \\
\hline
\end{tabular}

\section{Discussion}

Cette étude est une évaluation à partir de données hospitalières rétrospectives sur une période de trois années de la prévalence et les facteurs associés à la décompensation cétosique chez les patients diabétiques suivis au centre hospitalo-universitaire à Cotonou, au Bénin. Ce travail permet d'actualiser les données dix ans après une première étude réalisée dans le même centre.

Dans le présent travail, l’âge moyen des patients était de 43,7 ans \pm 16,2 ans. Ce résultat est superposable à celui retrouvé dans le même service il y a une décennie [Gninkoun et al. 2016] et à celui de KAKOMA et al. en 2014 [KAKOMA et al. 2014] en république démocratique du Congo. En revanche dans la série de LEYE et al. en 2016 au Sénégal [LEYE et al. 2016], les patients étaient légèrement plus âgés avec un âge moyen de 46,78 ans. Il apparaît donc que les décompensations cétosiques surviennent de façon générale chez les sujets relativement jeunes. Les sujets de sexe féminin étaient modestement prédominants dans notre travail comme déjà rapportés par certains auteurs [LEYE et al. 2016, Sarr A et al. 2011, Guira O et al. 2013], à contrario d'une étude réalisée au Benin en 2009 [Gninkoun et al. 2009] où il a été retrouvé une prédominance masculine. Dans une autre étude réalisée par DJROLO et al. [Djrolo et al. 2012] en 2012 sur la prévalence du diabète sucré aucune différence significative n’avait été retrouvée entre les deux sexes. 
Dans la littérature, il n’a pas été rapporté de façon formelle l'association du sexe à l'acidocétose.

Le taux de prévalence hospitalière des décompensations cétosiques reste élevé dans certains pays. En effet, la prévalence de 28,99 \% retrouvée dans notre travail est comparable aux données rapportées par différents auteurs. BAZIN et al.[Bazin et al. 2016] ont retrouvé en 2014 en France une fréquence de 30,1 \%, de même que LOKROU et al.[Lokrou A et al. 2014] en 2014 en Côte d'Ivoire qui rapportaient une fréquence de 30,7\%. Cette prévalence élevée des décompensations cétosiques dans ces études récentes citées pourrait s'expliquer probablement par la prévalence élevée et croissante du diabète dans le monde [ International Diabetes Federation, 2017].

Sur le plan des facteurs de décompensation, les infections et l'inobservance thérapeutique occupaient une place de choix comme ce fut le cas il y a dix ans dans le même service. Nos données sont comparables à la plupart des études réalisées en Afrique notamment au Sénégal en 2013 [LEYE et al. 2016] et en Côte d'Ivoire en 2014 [Lokrou A et al. 2014]. Néanmoins, certains auteurs avaient rapporté des fréquences légèrement inférieures à celles retrouvées dans ce travail. En 2014, en Inde, SUWARTO et al. [Suwarto et al. 2014] avaient noté que les infections représentaient les facteurs de décompensations dans 58,3 \% des cas. En Syrie, ALOURFI et al. [Alourfi et al. 2012] avaient rapporté une fréquence de $55 \%$ pour les infections. En ce qui concerne l'inobservance thérapeutique, KAKOMA et al.[Kakoma et al. 2014] en république démocratique du Congo avaient rapporté qu'elle était responsable des décompensations cétosiques dans 29,4\% des cas. NDEBELE et al.[Ndebele et al. 2018] en Afrique du Sud avaient retrouvé un résultat similaire avec $28 \%$ d'inobservance thérapeutique. Toutefois, des fréquences plus élevées ont été rapportées par certains auteurs. POUYE A. [Pouye et al. 2003] au Sénégal a rapporté 82,3\% de cas d'infection ; BALDE et al.[Baldé et al. 2007] en Guinée avaient noté que les erreurs de traitement étaient les principaux facteurs de décompensation dans 66\% des cas et RANDALL et al. [Randall et al. 2011] notèrent aux Etats Unis, que la mauvaise observance thérapeutique était responsable des décompensations cétosiques dans 68\% des cas.

Dans notre échantillon le foyer génito-urinaire étaient présents dans $23,30 \%$ des cas, suivi de celui respiratoire dans $18,04 \%$ des cas et cutané dans $15,03 \%$ des cas. Le paludisme était retrouvé dans 32,33\% des cas.

Les foyers infectieux restent invariables avec une répartition diversement rapportée dans la littérature. SARR et al.[Sarr et al. 2011] au Sénégal, UMPIERREZ et al. [Umpierrez et al 2002] aux USA avaient retrouvé une prédominance des infections urogénitales et broncho-pulmonaires. Les mêmes observations ont été faites dix ans plus tôt dans le même service au Bénin [Gninkoun et al. 2016]. La fréquence des infections urogénitales était 
significativement plus fréquente chez les femmes. Il s’agit d'une donnée bien rapportée dans la littérature. En effet, dans une étude dans le service en 2019 ayant porté sur les infections urinaires chez le patient diabétique [Gninkoun et al. 2019] les auteurs avaient rapporté une fréquence de l'infection urinaire de $62,2 \%$ chez les femmes contre 37,8 \% chez les hommes. Girard et al. [Girard et al. 2006] avaient retrouvé une fréquence d'infection urinaire de $84,6 \%$ chez les femmes contre $15,4 \%$ chez les hommes. La nette prédominance de l'infection urinaire chez les patientes diabétiques pourrait s'expliquer par certains facteurs physiologiques favorisants tels que la brièveté de l'urètre féminin et le déséquilibre du diabète.

La prédominance de l'étiologie infectieuse dans les régions africaines est due à la forte prévalence des maladies infectieuses souvent endémiques. Le paludisme, principale infection endémique a toujours une forte fréquence même après une décennie [Gninkoun et al. 2016]. LOKROU et al. [Lokrou et al 2014] en Côte d'Ivoire avaient aussi rapporté une fréquence similaire de cas de paludisme ( $33 \%$ ). Malgré les efforts de lutte, la présente étude a montré que la fréquence du paludisme était croissante pendant la période d'étude et représentait respectivement 19,00 \% entre 2016-2017, 21,00 \% entre 20172018 et 37,00 \% pour 2018-2019, et correspondait à une fréquence moyenne de 25,67 \% sur l'ensemble de la période d'étude. La fréquence moyenne de paludisme semble donc avoir été constante au Bénin. Cette évolution croissante du paludisme dans les facteurs de décompensation pourrait s'expliquer par la fréquence élevée des cas dans la population béninoise. En effet, selon l'annuaire des statistiques sanitaires de 2017 au Bénin le paludisme était la première affection rencontrée en consultation et représentait 30,5 \% des cas [Ministère de la santé du Bénin, 2017].

\section{Conclusion}

Ce travail a montré que la prévalence des décompensations cétosiques reste encore élevée. Les principaux facteurs de décompensation restent identiques dominés par les infections. Les foyers pulmonaire, génito-urinaire et le paludisme restent prédominant après la décennie. Une étude ultérieure des déterminants de l'infection tels que l'influence du climat pourrait être utile pour la prévention ciblée des décompensations.

\section{References:}

1. Alourfi Z, Homsi H. Precipitating factors, outcomes, and recurrence of diabetic ketoacidosis at a university hospital in Damascus. Avicenna Journal of Medicine. 2015;5(1): 11-15.

2. Baldé M.N, Barry A.Y, Diallo M.M, Kaké A, Bah D, Diallo A.O. Identification des facteurs de décompensation du diabète à Conakry en 
vue d'une éducation ciblée des patients. Diabetes and Metabolism. 2007;3:89.

3. Bazin A, Meillet L, Schillo F, Desmettre T, Borot S. Admissions aux urgences pour hypoglycémie ou décompensation hyperglycémique chez les patients diabétiques: un passage obligé? Médecine des Maladies Métaboliques. 2016;10(3):275-279.

4. Bonkoungou P, Lankoandé M, Guira O, Saloukou KE, kaboré RA, Zinsou $\mathrm{A}$ et al. Complications aiguës métaboliques du diabète sucré dans le Service de Réanimation Polyvalente du Centre Hospitalier Universitaire Yalgado Ouédraogo de Ouagadougou au Burkina Faso. Revue Africaine et Malgache de Recherche Scientifique/Sciences de la Santé. 2017;5(1): 36-41.

5. Djrolo F, Houinato D, Gbary A, Akoha R, Djigbénoudé O, Sègnon J. Prévalence du diabète sucré dans la population adulte à Cotonou, Bénin. Médecine des maladies Métaboliques. 2012;6(2):167-169.

6. Djrolo F, Adoukonou T, Houehanou C, Houinato JD, Houinato D. Diabetes in Borgou Department in Benin: Prevalence and associated factors. Journal of Diabetes Mellitus. 2015;5(02):90 6.

7. Girard R, de Montclos M, Bournaud C, Orgiazzi J. Dépistage des bactériuries à l'admission chez les patients diabétiques : peut-on abandonner les examens cytobactériologiques urinaires systématiques? Médecine et maladies infectieuses 2006 ;36 ; 219-222.

8. Gning SB, Thiam M, Fall F, Ba-Fall K, Mbaye PS, Fourcade L. Le diabete sucre en Afrique subsaharienne. Aspects epidemiologiques, difficultes de prise en charge. Médecine tropicale. 2007;67(6):607 11.

9. Gninkoun CJ, Mushaniko-Bita D, Alassani SCA, Sylla SD, Dedjan $\mathrm{AH}$. Infection urinaire chez le patient diabétique à Cotonou : aspects épidémiologiques et facteurs associés. Journal de la Société de Biologie Clinique du Bénin, 2019 ; 032 : 126-130

10. Gninkoun CJ, Alassani AS, Sagna Y, Adjagba P, Djrolo F. Diabetic Ketosis Decompensations at the National Hospital in Benin (West Africa), What Did We Learn about the Precipitating Factors? Journal of Diabetes Mellitus. 2016;6(04):301 6.

11. Guira O, Tiéno H, Bagbila A, Sagna Y, Nikiéma P, Yanogo D, et al. Typologie des cétoses diabétiques et facteurs associés aux dysnatrémies à la phase initiale de leur prise en charge à Ouagadougou (Burkina Faso). Médecine des Maladies Métaboliques. 2013;7(6):539-542.

12. International Diabetes Federation. Atlas du diabète. 8è Edition. 2017;138:271 81.

13. Kakoma PK, Kadiebwe DM, Kayembe AM, Makonga PK, Bugeme M, Mukuku O. Acidocétose diabétique chez l'adulte à l'Hôpital 
Sendwe de Lubumbashi: à propos de 51 cas. Pan African Medical Journal. 2014;17(1):1-5.

14. Leye MY, Leye A, Ndiaye N, Ngo VB, Touré PS, Ndour M, Fall B. Aspects épidémiologiques et diagnostiques de la cétoacidose diabétique en milieu hospitalier à Dakar. Analyse de 102 cas au CHU de Pikine. Revue Africaine de Médecine Interne. 2016;3(2):8 11.

15. Lokrou A, Kouassi F. Bilan de 9 années de prise en charge de l'acidocétose du diabétique africain adulte en Côte-d'Ivoire. Médecine des maladies métaboliques. 2014;8(3):330-334.

16. Ministère de la santé du Bénin. Annuaire des statistiques sanitaires 2017. Bénin; 2017. 135.

17. Ndebele NFM, Naidoo M. The management of diabetic ketoacidosis at a rural regional hospital in KwaZulu-Natal. Afr J Prim Health Care Fam Med. 2018; 10(1): 1612.

18. Pouye A, Leye A, Ndongo S, Ka MM, Dia D, Fall S, et al. Acidocétose diabétique dans un service de Médicine interne. Dakar médical 2003 ; 48:108-111.

19. Randall L, Begovic J, Hudson M, Smiley D, Peng L, Pitre N, et al. Recurrent diabetic ketoacidosis in inner-city minority patients: behavioral, socioeconomic, and psychosocial factors. Diabetes care. 2011;34(9):1891-1896.

20. Sarr A, Diedhiou D, Ndour-Mbaye N.M, Leye Y.M, Ka-Cisse M.S, Leye A, et al. Ketoacidosis in type 1 diabetes mellitus: 73 cases in Dakar. Le Mali medical. 2011;26(4):50-54.

21. Suwarto S, Sutrisna B, Waspadji S, Pohan H.T. Predictors of five days mortality in diabetic ketoacidosis patients: a prospective cohort study. Acta Medica Indonesiana. 2014;46(1): 18-23.

22. Tchaou BA, Gomina M, Agbo AHM, Akpona SA. Complications aiguës métaboliques du diabète sucré dans l'Unité de réanimation de l'Hôpital Universitaire de Parakou (Bénin). European Scientific Journal, ESJ. 2014;10(24): 208-218.

23. Umpierrez GE, Murphy MB, Kitabchi AE. Diabetic Ketoacidosis and Hyperglycemic Hyperosmolar Syndrome. Diabetes Spectrum. 2002;15(1):28 36.

24. Wild S, Roglic G, Green A, Sicree R, King H. Global prevalence of diabetes: estimates for the year 2000 and projections for 2030. Diabetes care. 2004; 27(5):1047 53. 